\title{
PAPER
}

\section{Heart rate dynamics in refractory and well controlled temporal lobe epilepsy}

\author{
H Ansakorpi, J T Korpelainen, H V Huikuri, U Tolonen, V V Myllylä, J I T Isojärvi
}

J Neurol Neurosurg Psychiatry 2002;72:26-30

See end of article for authors' affiliations

....................

Correspondence to: Dr H Ansakorpi, Department of Neurology, University of Oulu, Box 5000, Fin-90014,

University of Oulu, Finland; htansakorpi@hotmail.com

Received

19 January 2001

In revised form 25 June

2001

Accepted 6 July 2001

Objectives: Disorders of cardiovascular and other autonomic nervous system functions are often found in patients with temporal lobe epilepsy (TLE). Cardiovascular dysregulation in TLE has previously been quantified assessing traditional time and frequency domain measures of heart rate (HR) variability from short term ECG recordings. However, new complexity and fractal measures of HR variability based on non-linear dynamics and fractals ("chaos theory") may disclose certain patterns of HR dynamics that cannot be detected using only conventional measures.

Methods: In addition to the traditional spectral and non-spectral components of HR variability, fractal correlation properties, approximate entropy (ApEn) of RR interval dynamics, and the slope of the power law relation were measured from 24 hour ambulatory ECG recordings to evaluate interictal autonomic cardiovascular regulatory function in 19 patients with refractory TLE, 25 patients with well controlled TLE, and in 34 healthy age and sex matched control subjects.

Results: The traditional time and frequency domain measures were lower in patients with TLE than in controls $(p<0.05)$. In addition, the power law slope $(p<0.005)$ and $A p E n(p<0.05)$ were also reduced in TLE patients. Furthermore, ApEn was smaller in patients with refractory TLE than in patients with wellcontrolled TLE ( $p<0.01)$, whereas the long term fractal correlation value $\alpha 2$ was lower in patients with well controlled TLE $(p<0.05)$. An altered HR variation was not associated with any particular AED regimen.

Conclusions: In addition to reduced overall HR variability, the long term fractal organisation and complexity of HR dynamics seem to be altered in TLE. These abnormalities in HR behaviour may partly contribute to the occurrence of adverse cardiovascular events, such as life threatening arrhythmias in patients with TLE.

S udden unexpected death in epilepsy (SUDEP) is an intriguing phenomenon, yet its basic mechanisms still remain unknown. There are known risk factors for SUDEP $^{1-6}$ but otherwise healthy, compliant patients may also die suddenly. ${ }^{4}$ SUDEP is thought to be a peri-ictal event, ${ }^{3-5} 7$ and it seems apparent that the autonomic cardiac regulatory system is involved, either by ventilatory or by cardiovascular dysfunction. ${ }^{7-10}$ Moreover, patients with carbamazepine have been suggested to be overrepresented among subjects experiencing SUDEP. ${ }^{11}$

Ictal changes in heart rate (HR), blood pressure (BP), and other autonomic functions have been described in detail in patients with epilepsy, ${ }^{12}$ but little is known about the possible interictal alterations of cardiovascular autonomic regulation. Previous studies, based on short term ECG recordings under laboratory conditions or ambulatory ECG recordings suggest that HR variability is reduced in temporal lobe epilepsy (TLE) ${ }^{13-18}$ Reduced HR variability, on the other hand, is well known to be associated with increased susceptibility to cardiac arrythmias. ${ }^{19-28}$ However, it has been difficult to distinguish the roles of the epileptic process itself and antiepileptic drugs (AEDs) in modifying cardiac regulation in patients with TLE.

Analysis of HR variability is the most commonly used measure of the cardiovascular autonomic regulatory system. Power spectrum analysis of HR variability from ambulatory ECG recordings provides a tool for quantitatively assessing the effects of the autonomic nervous system on the heart. Recently new methods based on non-linear dynamics and fractal analysis ("chaos theory") have been developed to quantify complex HR dynamics and to complement conventional spectral measures of HR variability. ${ }^{29-32}$ They have already provided clinically useful information on cardiac diseases, ${ }^{19-2125-2633}$ but they have not been used in the evaluation of autonomic cardiovascular dysfunction in epilepsy so far.

The aim of this study was to evaluate the function of the cardiovascular autonomic regulatory system in patients with TLE by using various measurements of traditional spectral and non-spectral components of HR fluctuation as well as complexity and fractal measures of HR variability. In addition, we aimed to determine whether reduced HR variability is associated with the severity of TLE or certain AED regimens.

\section{PATIENTS AND METHODS}

\section{Patients and control subjects}

The study was carried out at the Outpatient Department of Neurology, Oulu University Hospital, Finland, with the approval of the ethics committee of the Medical Faculty of University of Oulu.

Forty four consecutive patients with TLE seen in the outpatient clinic participated in the study after giving their informed consent. Female patients who were pregnant or lactating were excluded. Similarly, symptoms or signs of illness other than epilepsy (for instance diabetes mellitus, cardiopulmonary diseases, alcoholism, or renal failure) or medication known to affect the autonomic nervous system were used as exclusion criteria. In general, the patients had neither excessive body weight, nor increased BP, and laboratory screening (liver and renal function, serum electrolytes, basic haematological indices) was normal in all the study patients.

Abbreviations: TLE, temporal lobe epilepsy; SUDEP, sudden unexpected death in epilepsy; HR, heart rate; ApEn, approximate entropy; BP, blood pressure; AEDs, antiepileptic drugs; 
Table 1 Demographics of the study patients and the control subjects

\begin{tabular}{lcll}
\hline & $\begin{array}{l}\text { No of } \\
\text { patients }\end{array}$ & $\begin{array}{l}\text { Age }(y, \\
\text { mean } \\
\text { (SD)) }\end{array}$ & $\begin{array}{l}\text { Duration of } \\
\text { epilepsy }(y, j \\
\text { mean (SD)) }\end{array}$ \\
\hline $\begin{array}{l}\text { Patients with refractory TLE: } \\
\text { Male }\end{array}$ & 5 & $29.8(7.9)$ & $15.0(8.8)$ \\
Female & 14 & $33.9(6.5)$ & $24.3(10.0)$ \\
$\quad \begin{array}{l}\text { Total } \\
\text { Patients with well controlled TLE: }\end{array}$ & $19.8(7.0)$ & $21.8(10.4)$ \\
$\begin{array}{l}\text { Male } \\
\text { Female }\end{array}$ & 11 & $30.5(5.8)$ & $16.6(10.5)$ \\
Total & 14 & $35.4(5.9)$ & $15.3(9.9)$ \\
Control subjects: & 25 & $33.3(6.2)$ & $15.9(10.0)$ \\
Male & 9 & $28.1(6.2)$ & \\
Female & 25 & $35.2(10.2)$ & \\
Total & 34 & $33.3(9.7)$ & \\
\hline
\end{tabular}

Consecutive patients seen in the outpatient clinic were considered for the study and the exclusion criteria were met more often in male patients than in female patients, which resulted in fewer male patients in the study.

All patients were carefully interviewed and clinically examined and the epilepsy type was classified according to the recommendations of the International League Against Epilepsy. ${ }^{34}$ Nineteen patients had refractory TLE with recurrent monthly seizures despite the regular use of adequate AEDs whereas 25 patients had well controlled TLE and most of them had been seizure free since starting the antiepileptic treatment. The demographics of the study patients and control subjects are given in table 1 . The AEDs taken by the patients are shown in tables 2 and 3.

Interictal EEG recording was obtained from all the patients. Normal EEG or general slowing was seen in 15 patients. Left temporal focal slow waves, or irritation, or both were detected in 17 patients, whereas right temporal focal disturbances were seen in nine patients. One patient had bilateral temporal focal irritation.

Magnetic resonance imaging was performed in all except three patients with well controlled TLE who had claustrophobia. Computed tomography was performed in those three patients. Four patients had left hippocampal sclerosis whereas three patients had right hippocampal sclerosis. One patient had left hippocampal sclerosis and parahippocampal cortical dysplasia. All the other patients had normal imaging of the brain.

The control group consisted of 34 healthy age and sex matched subjects selected from healthy people who were participating in a trial comparing characteristics of hypertensive and normotensive subjects randomly selected by social security code from the general population of Oulu. They all underwent a complete physical examination and had no disease or medication affecting the autonomic nervous system in their medical history.

\section{Methods}

A two channel 24 hour ambulatory ECG recording (Delmar Avionics electroscanner) was performed in all the patients and control subjects. ${ }^{35}$ During the recording they continued to perform their normal daily activities. They were also asked to keep a diary of all the events and possible seizures during the recording.

The ECG data from the recordings were sampled digitally and transferred from the Oxford Medilog scanner to a microcomputer for analysis of HR variability. All RR interval time series were first edited automatically, after which careful manual editing was performed by visual inspection of the RR intervals. Each RR interval time series was passed through a filter that eliminates premature beats and artifacts and deletes the filling gaps as previously described. ${ }^{23-25}$

Table 2 Antiepileptic medication of the study patients

\begin{tabular}{|c|c|c|c|c|c|c|}
\hline & \multicolumn{3}{|c|}{ Refractory TLE } & \multicolumn{3}{|c|}{ Well controlled TLE } \\
\hline & Male & Female & Total & Male & Female & Total \\
\hline CBZ & - & - & - & 6 & 8 & 14 \\
\hline OXC & - & 4 & 4 & 4 & 4 & 8 \\
\hline PHT & - & - & - & - & 1 & 1 \\
\hline VPA & 1 & - & 1 & - & - & - \\
\hline LTG & - & - & - & - & 1 & 1 \\
\hline CBZ with other $A E D(s)$ & 2 & 5 & 7 & 1 & - & 1 \\
\hline OXC with other AED(s) & 2 & 5 & 7 & - & - & - \\
\hline
\end{tabular}

CBZ, carbamazepine; OXC, oxcarbazepine; LTG, lamotrigine; VPA, valproate; PHT, phenyłoin; CZP, clonazepam.

Table 3 Daily doses and serum concentrations of the antiepileptic drugs (AEDs) in study patients

\begin{tabular}{|c|c|c|c|}
\hline AED & No of patients & Dose (mg) & Serum concentration (M) \\
\hline \multicolumn{4}{|l|}{ Patients with refractory TLE: } \\
\hline OXC & 4 & $1613(448)$ & $69.4(11.1)^{*}$ \\
\hline VPA & 1 & 2500 & 0.56 \\
\hline CBZ with other AEDs & 7 & $1057(230)$ & $29.9(7.4)$ \\
\hline OXC with other AEDs & 7 & $1586(334)$ & $83.8(13.3)$ \\
\hline \multicolumn{4}{|c|}{ Patients with well controlled TLE: } \\
\hline CBZ & 14 & $614(160)$ & $28.8(6.7)$ \\
\hline OXC & 8 & 975 (311) & $48.4(18.9)$ * \\
\hline PHT & 1 & 200 & 38.9 \\
\hline LTG & 1 & 400 & 8.83 \\
\hline CBZ with other AEDs & 1 & 700 & 21.0 \\
\hline
\end{tabular}

*Monohydroxy carbamazepine, the active metabolite of OXC

CBZ, carbamazepine; OXC, oxcarbazepine; VPA, valproate; PHT, phenytoin; LTG, lamotrigine. 


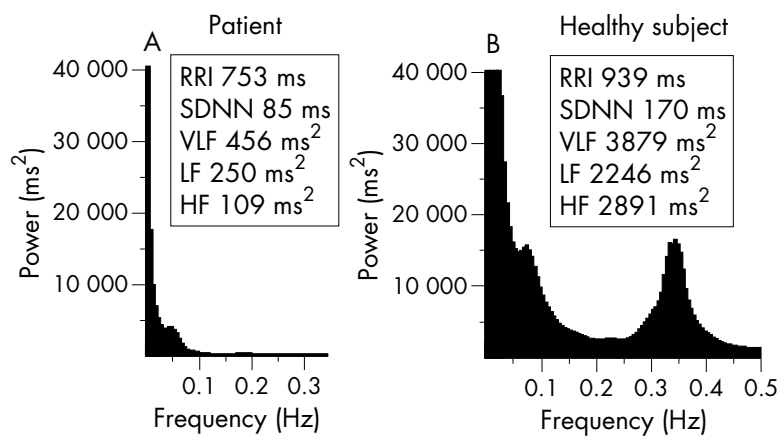

Figure 1 Power spectral analysis of HR variability $(A)$ in a 35 year old male patient with TLE and (B) in a healthy 27 year old male control subject. The area under the spectral curve from 0.005 to $0.04 \mathrm{~Hz}$ represents VLF power, the area from 0.04 to 0.15 represents LF power, and the area from 0.15 to $0.4 \mathrm{~Hz}$ represents HF power. The patient withTLE shows a typical suppression of all the power spectral components of HR variability. RRI indicates RR interval.
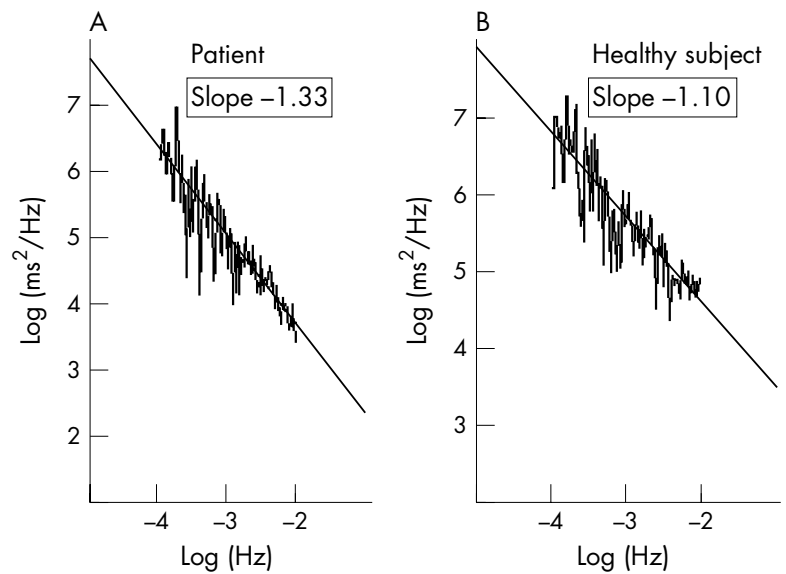

Figure 2 Log-log graphs of 24 hour RR interval spectra from (A) a 35 year old male patient with TLE and (B) a healthy 27 year old male control subject. The patient with TLE shows a deeper curve of the slope.

In the final analysis of linear components of HR variability, 24 hour measurements were divided into segments of $3600 \mathrm{RR}$ intervals, and in the analysis of non-linear components of HR variability 24 hour measurements were divided into segments of 8000 and only segments with more than $85 \%$ sinus beats were included. The mean length of all RR intervals and SD of all RR intervals (SDNN) were computed as time domain measures. The power spectra of HR variability (fig 1) were quantified by measuring the area in three frequency bands: 0.005 to $0.04 \mathrm{~Hz}$ (very low frequency, VLF), 0.04 to $0.15 \mathrm{~Hz}$ (low frequency, LF), and 0.15 to 0.4 (high frequency, HF).

ApEn, a measure that quantifies the regularity of time series data, was calculated from 24 hour recordings using a previously described method. ${ }^{19}{ }^{2631}$ To quantify fractal correlation properties of HR, the detrended fluctuation analysis technique, which is a modified root mean square analysis of random walk, was used. ${ }^{26}{ }^{36}{ }^{37}$ In this study, HR correlation properties were defined separately for short term $(\leqslant 11$ beats, $\alpha 1)$ and for long term (>11 beats, $\alpha 2$ ) correlations of RR interval data (short and long term scaling exponents). ${ }^{263637}$ The power law relation of RR interval variability was calculated from the frequency range of $10^{-4}$ to $10^{-2}$ by a recently described method (fig 2). ${ }^{38}{ }^{39}$

Statistical analyses were performed using the KruskallWallis test and the Mann-Whitney two sample test to compare the values of the control subjects and those of the patients.
Correlations between ApEn, the slope of the power law relation of the HR variability and the traditional spectral measures of HR variation in patients with TLE were assessed with Pearson's bivariate correlation test.

\section{RESULTS}

Six patients with refractory TLE reported having experienced partial seizures during the recording. Their HR variation profile did not, however, show any particularly different pattern of HR variation profiles compared with the other patients and they were, therefore, included in the analysis.

The mean value of the RR interval $(p<0.403)$ was similar in the patients and the control subjects. The mean values of the different measures of HR variability in patients and in control subjects are presented in table 4 . In patients with TLE, the values of SDNN $(p<0.05)$, and the spectral components VLF $(\mathrm{p}<0.001), \operatorname{LF}(\mathrm{p}<0.001)$ and $\operatorname{HF}(\mathrm{p}<0.01)$ were lower compared with those of the control subjects. The value of ApEn $(p<0.05)$ was also diminished in patients with TLE and the slope of the power law relation of the HR variability was also deeper $(p<0.005)$ in the patient group than in the control subjects. The fractal measures of HR variability did not show any differences between the patients and the control subjects.

The mean value of the ApEn of the refractory patients with TLE was lower $(p=0.021)$ than that of the well controlled TLE and the long term fractal correlation value $\alpha 2$ was smaller $(p=0.037)$ in patients with well controlled TLE. All other values of the HR measures among patients with refractory and well controlled TLE were similar (table 4).

Altered HR variability was not associated with any particular AED regimen, nor did serum concentrations of different AEDs have any effect on the HR variability. Moreover, the duration of TLE did not correlate with the magnitude of HR variability, and the mean values of ApEn and the slope of the power law relation of the HR variability did not correlate with the traditional spectral measures in patients with TLE, either.

\section{DISCUSSION}

In the present study, not only the traditional time and frequency domain measures of HR variability, but also ApEn and the slope of the power law relation of HR variability were suppressed in patients with TLE reflecting cardiovascular autonomic dysfunction. Based on these results it seems that in addition to the conventional measures of HR variability, new dynamic measures not related to means and variance are useful in detecting altered HR behaviour in patients with TLE.

Altered HR variability has previously been described in patients with epilepsy by using power spectral analysis. ${ }^{15} 16$ However, the methods and variables used as well as study designs are different in the various studies, and, therefore, the results are difficult to compare. To our knowledge, this is the first study to evaluate the association between altered HR variability and the severity of TLE. Moreover, nonconventional measures that are able to describe long term tonic oscillations of HR variability have not been previously used to evaluate autonomic dysfunction in TLE.

The pathophysiological basis of the cardiovascular autonomic dysfunction in TLE is not completely understood. The insular cortex is considered to be the most important cortical area controlling cardiovascular regulation, and it has extensive connections with other cardiovascular control centres. ${ }^{40-42}$ In TLE, the epileptic focus is close to these frontal and temporal regulatory areas and may interfere with their function. Interestingly, in the present study the changes in cardiovascular regulation were not associated with the severity of TLE or any particular AED regimen, but they were seen in both well controlled and refractory patients, as well as in patients taking different AEDs. Therefore, according to the present results, TLE itself may be implicated. 
Table 4 Heart rate and measures of heart rate variability in patients with TLE and control subjects

\begin{tabular}{llllll}
\hline $\begin{array}{l}\text { Measure/ } \\
\text { variable }\end{array}$ & $\begin{array}{l}\text { Patients with } \\
\text { refractory TLE } \\
(\mathrm{n}=9)\end{array}$ & $\begin{array}{l}\text { Patients with } \\
\text { well controlled } \\
\text { TLE }(\mathrm{n}=25)\end{array}$ & $\begin{array}{l}\text { All patients } \\
(\mathrm{n}=44)\end{array}$ & $\begin{array}{l}\text { Control subjects } \\
(\mathrm{n}=4)\end{array}$ & $\begin{array}{l}\text { p Valuef } \\
\text { (Mann-Whitney) }\end{array}$ \\
\hline RRI (ms) & $863(103)$ & $819(112)$ & $838(109)$ & $850(71)$ & 0.403 \\
SDNN (ms) & $154(32)$ & $158(51)$ & $156(43)$ & $177(44)$ & 0.039 \\
VLF $\left(\mathrm{ms}^{2}\right)$ & $1538(762)$ & $1749(1005)$ & $1658(905)$ & $3256(1765)$ & $<0.001$ \\
LF $\left(\mathrm{ms}^{2}\right)$ & $905(472)$ & $1038(507)$ & $981(491)$ & $1901(1308)$ & 0.001 \\
$\mathrm{HF}\left(\mathrm{ms}^{2}\right)$ & $604(502)$ & $628(365)$ & $618(424)$ & $1710(2050)$ & 0.011 \\
ApEn & $0.93(0.21) \dagger$ & $1.09(0.32)$ & $1.02(0.29)$ & $1.15(0.21)$ & 0.045 \\
$\alpha 1$ & $1.22(0.18)$ & $1.17(0.18)$ & $1.19(0.18$ & $1.16(0.1)$ & 0.292 \\
$\alpha 2$ & $1.03(9.0)$ & $0.99(7.64)^{*}$ & $1.01(8.45)$ & $1.01(6.13)$ & 0.824 \\
Slope of HRV & $-1.32(0.13)$ & $-1.37(0.21)$ & $-1.35(0.18)$ & $-1.20(0.21)$ & 0.002
\end{tabular}

Values are mean (SD); RRI, R-R interval; SDNN, SD of all RRIs; VLF, very low frequency; LF, low frequency; $\mathrm{HF}$, high frequency; ApEn, approximate entropy; $\alpha 1$, short term scaling exponent; $\alpha 2$, long-term scaling exponent; ${ }^{*} p=0.037$ compared with the patients with refractory TLE; $\nmid p=0.021$ compared to the patients with well controlled TLE; $\ddagger$ all patients compared with the control subjects.

Although contrary to some earlier studies, ${ }^{16-18} 43$ AEDs did not seem to differ with regard to their propensity of modifying HR variation, this does not exclude the possibility that AEDs could have effects on cardiovascular regulatory function. All AEDs taken by the patients in the present study act by blocking sodium channels as do many antiarrhythmic agents-for instance, those which are associated with increased arrhythmogenic mortality. ${ }^{44}$ Therefore, AEDs may have effects on the cardiac conduction system as well as on centrally mediated cardiovascular control system function and contribute to the changes. However, the present results do not support the view that carbamazepine has a more potent effect on the cardiac regulatory system than the other commonly used AEDs. ${ }^{111} 16-184346$

Traditional spectral and non-spectral measurements have proved useful in determining autonomic regulatory dysfunction in various clinical conditions. ${ }^{27}$ The physiological and clinical applicability of fractal and complexity measures of HR dynamics are not yet completely understood, but they are suggested to show abnormal patterns of RR interval behaviour that are not easily detected by commonly used moment statistics of HR variation. ${ }^{19}$ Reduced ApEn indicates larger predictability in HR behaviour and it has previously been described to correlate with various pathological conditions-for example, risk of the sudden infant death syndrome. ${ }^{19} 31$ 47-49

Reduced ApEn has been found to be associated with the onset of paroxysmal atrial fibrillation in subjects without any structural heart disease ${ }^{28}$ and infusion of noradrenaline, ${ }^{50}$ possibly reflecting concomitant, accentuated sympathetic and vagal outflow to the sinus node. Interestingly, in the present study, ApEn was significantly lower in patients with refractory TLE than in patients with well controlled TLE. This may result from increased serum concentrations of noradrenaline induced by frequent seizures. The mean values of ApEn and the slope of the power law relation of HR variability did not correlate with the traditional spectral measures of HR variability, showing that these new indices may be more sensitive than conventional methods in detecting altered cardiovascular regulation in patients with TLE.

Similar to our findings in patients with TLE, altered autonomic cardiovascular function has also been described in various cardiac diseases. Several studies have shown that reduced HR variability is an independent risk factor for arrhythmic sudden death after myocardial infarction. ${ }^{25} 263840$ recent study on a large number of elderly people also showed that the slope of the power law relation of HR variability most strongly predicts cardiovascular mortality in a general population of elderly subjects. ${ }^{22}$ Therefore, it is possible that changes in these variables may also predict the cardiovascular risk in patients with TLE.

In summary, HR variability is altered in patients with TLE and the reduced HR variability can be quantified by using both traditional and dynamic measures of HR variability. The altered cardiovascular regulation seems to be related to the epileptic process itself rather than to the severity or duration of TLE or to any specific AED regimen, although ApEn of the complexity measures seems to be lower in patients with refractory TLE than in patients with well controlled TLE. Methods used in this study may be useful in evaluating abnormal HR dynamics in patients with TLE. However, further studies are needed to assess the association between altered HR dynamics and risk for sudden death in patients with TLE.

\section{Authors' affiliations}

H Ansakorpi, J T Korpelainen, H V Huikuri, U Tolonen, V V Myllylä, J I T Isoiärvi, Departments of Neurology and Medicine, Division of Cardiology, University of Oulu, Oulu, Finland

\section{REFERENCES}

1 Devinsky O, Perrine K, Theodore WH. Interictal autonomic nervous system function in patients with epilepsy. Epilepsia 1994;35:199-204.

2 Tennis P, Cole TB, Annegers JF, et al. Cohort study of incidence of sudden unexplained death in persons with seizure disorder treated with antiepileptic drugs in Saskatchewan, Canada. Epilepsia 1995:36:29-36.

3 Leestma JE, Annegers JF, Brodie M, et al. Sudden unexplained death in epilepsy: observations from a large clinical development program. Epilepsia 1997;38:47-55

4 Nashef L, Garner S, Sander JWAS, et al. Circumstances of death in sudden death in epilepsy: interviews of bereaved relatives. J Neurol Neurosurg Psychiatry 1998:64:349-52.

5 Nilsson L, Farahmand BY, Persson P-G, et al. Risk factors for sudden unexpected death in epilepsy: a case-control study. Lancet 1999;353:888-93.

6 Sperling MR, Feldman $\mathrm{H}$, Kinman J, et al. Seizure control and mortality in epilepsy. Ann Neurol 1999:46:45-50.

7 Johnston SC, Siedenberg R, Min JK, et al. Central apnea and acute cardiac ischemia in a sheep model of epileptic sudden death. Ann Neurol 1997;42:588-94

8 Johnston CJ, Horn JK, Valente J, et al. The role of hypoventilation in a sheep model of epileptic sudden death. Ann Neurol 1995;37:531-7.

9 Nashef L, Walker F, Allen P, et al. Apnoea and bradycardia during epileptic seizures: relation to sudden death in epilepsy. J Neurol Neurosurg Psychiatry 1996;60:297-300.

10 Schraeder PL, Lathers CM. Paroxysmal autonomic dysfunction, epileptogenic activity and sudden death. Epilepsy Res 1989:3:55-62.

11 Timmings PL. Sudden unexpected death in epilepsy: is carbamazepine implicated? Seizure 1998;7:289-91.

12 Wannamaker BB. Autonomic nervous system and epilepsy. Epilepsia 1985;26(suppl 1):S31-9.

13 Messenheimer JA, Quint SR, Tennison MB, et al. Monitoring heart rate period variability changes during seizures. I. Methods. J Epilepsy 1990;3:47-54

14 Frysinger RC, Engel J, Harper RM. Interictal heart rate patterns in partia seizure disorders. Neurology 1993;43:2136-9.

15 Massetani $\mathbf{R}$, Strata $G$, et al. Alteration of cardiac function in patients with temporal lobe epilepsy: different roles of EEG-ECG monitoring and spectral analysis of RR variability. Epilepsia 1997;38:363-9.

16 Tomson T, Ericson M, Ihrman C, et al. Heart rate variability in patients with epilepsy. Epilepsy Res 1998;30:77-83.

17 Isoiärvi JIT, Ansakorpi H, Suominen K, et al. Interictal cardiovascular responses in patients with epilepsy. Epilepsia 1998;39:420-6. 
18 Ansakorpi $\mathbf{H}$, Korpelainen JT, Suominen $\mathrm{K}$, et al. Interictal cardiovascular autonomic responses in patients with temporal lobe epilepsy. Epilepsia 2000;41:42-7.

19 Mäkikallio TH, Seppänen T, Niemelä M, et al. Abnormalities in beat to beat complexity of heart rate dynamics in patients with a previous myocardial infarction. J Am Coll Cardiol 1996;28:1005-11.

20 Brouwer J, van Veldhuisen DJ, Man in't Veld AJ. Prognostic value of heart rate variability during long-term follow-up in patients with mild to moderate heart failure. J Am Coll Cardiol 1996;28:1 183-9.

21 Ho KKL, Moody GB, Peng CK, et al. Predicting survival in heart failure cases and controls using fully automated methods for deriving nonlinear and conventional indices of heart rate dynamics. Circulation 1997;96:842-8

22 Huikuri HV, Mäkikallio TH, Airaksinen KEJ, et al. Power-law relationship of heart variability as a predictor of mortality in the elderly. Circulation 1997:96:842-8

23 Huikuri HV, Valkama JO, Airaksinen KEJ, et al. Frequency domain measures of heart rate variability before onset of non-sustained and sustained ventricular tachycardia in patients with coronary artery disease. Circulation 1993:87:1220-8.

24 Huikuri HV, Niemelä M, Ojala S, et al. Circadian rhythms of frequency domain measures of heart rate variability in healthy subjects and patients with coronary artery disease: effects of arousal and upright posture. Circulation 1994;90:121-6.

25 Huikuri HV, Seppänen T, Koistinen M, et al. Abnormalities in beat-to-beat dynamics of heart rate before the spontaneous onset of life-threatening ventricular tachyarrhythmias in patients with prior myocardial infarction. Circulation 1996;93:1836-44.

26 Mäkikallio TH, Seppänen T, Airaksinen KEJ, et al. Dynamic analysis of heart rate may predict subsequent ventricular tachycardia after myocardial infarction. Am J Cardiol 1997;80:779-83.

27 Huikuri HV. Heart rate variability in coronary artery disease. J Intern Med 1995;237:349-57.

28 Vikman S, Mäkikallio TH, Yli-Mäyry S, et al. Altered complexity and correlation properties of R-R interval dynamics before the spontaneous onset of paroxysmal atrial fibrillation. Circulation 1999;100:2079-84.

29 Goldberger AL, West BJ. Applications of non-linear dynamics to clinical cardiology. Ann N Y Acad Sci 1987;504:155-212.

30 Denton TA, Diamond GA, Helfant RH, et al. Fascinating rhythm: a primer on chaos theory and it applications to cardiology. Am Heart J 1990;120:1419-39.

31 Pincus SM, Goldberger AL. Physiologic time-series analysis: what does regularity quantify? Am J Physiol 1994;226:H1643-56

32 Goldberger AL. Non-linear dynamics for clinicians: chaos theory, fractals, and complexity at bedside. Lancet 1996:347:1312-14.

33 Woo MA, Stevenson WG, Moser DK, et al. Complex heart rate variability and serum norepinephrine levels in patients with advanced heart failure. J Am Coll Cardiol 1994;23:565-9.

34 Proposal for revised classification of epilepsies and epileptic syndromes: from the Commission on Classification and Terminology of the International League Against Epilepsy. Epilepsia 1989;30:389-99.
35 Heart rate variability. Standards of measurement, physiological interpretation, and clinical use. Electrophysiology. Circulation 1996;93:1043-65.

36 Peng CK, Havlin S, Stanley HE, et al. Quantification of scaling exponents and crossover phenomena in non-stationary heartbeat time series. Chaos 1995;1:82-7.

37 lyengar N, Peng CK, Ladin Z, et al. Age-related alterations in the fractal scaling of cardiac interbeat interval dynamics. Am J Physio 1996;271:R1078-84.

38 Bigger JT Jr, Steinman RC, Rolnitzky LM, et al. Power law behaviour of RR-interval variability in healthy middle-aged persons, patients with recent acute myocardial infarction, and patients with heart transplants. Circulation 1996:93:2142-51.

39 Saul JP, Albrecht P, Berger RD, et al. Analysis of long-term heart rate variability: methods, $1 / f$ scaling and implications. In: Computers in cardiology. Silver Spring. MD: IEEE Computer Society Press; 1987:419-22.

40 Oppenheimer S. The anatomy and physiology of cortical mechanisms of cardiac control. Stroke 1993;24(suppl I):I3-5.

41 Cechetto DF, Saper CB. Role of the cerebral cortex in autonomic function. In: Loewy AD, Spyer KN, eds. Central regulation of autonomic functions. New York: Oxford University Press, 1990:208-23.

42 Cechetto DF. Neuropathology and cardiovascular regulation. In: Horst GJT, ed. The nervous system and the heart. New Jersey: Humana Press, 2000: 159-79

43 Quint SR, Messenheimer JA, Tennison MB. Power spectral analysis: a procedure for assessing autonomic activity related to risk factors for sudden and unexplained death in epilepsy. In: Lathers CM, Schraeder PL, eds. Epilepsy and sudden death. New York: Marcel Dekker, 1990:261-91.

44 The Cardiac Arrhythmias Suppression Trial (CAST) investigators. Preliminary report: effect of encainide and flecainide on mortality in a randomized trial of arrhythmia suppression after myocardial infarction. $N$ Engl J Med 1989;321:406-12.

45 The Cardiac Arrhythmias Suppression Trial II Investigators. Effect of the antiarrhythmic agent moricine on survival after myocardial infarction. N Engl J Med 1992;327:227-33.

46 Tomson T, Kennebäck G. Arrhythmia, heart rate variability, and antiepileptic drugs. Epilepsia 1997;38(suppl 11): S48-51.

47 Pincus SM. Approximate entropy as a measure of system complexity. Proc Natl Acad Sci 1991;88:2297-301.

48 Pincus SM, Viscarello RR. Approximate entropy: a regularity statistic for fetal heart rate analysis. Obstet Gynecol 1992;79:249-55.

49 Fleisher LA, Pincus SM, Rosenbaum SH. Approximate entropy of heart rate as a correlate of postoperative ventricular dysfunction. Anesthesiology 1993;78:683-92.

50 Tulppo M, Mäkikallio TH, Airaksinen KEJ, et al. Physiological correlates of complexity of R-R interval dynamics [abstract]. Eur Heart J 1999;20(suppl): 1-753. 